\title{
UNBOUNDED COMMUTING OPERATORS AND MULTIVARIATE ORTHOGONAL POLYNOMIALS
}

\author{
YUAN XU
}

(Communicated by Palle E. T. Jorgensen)

\begin{abstract}
The multivariate orthogonal polynomials are related to a family of operators whose matrix representations are block Jacobi matrices. A sufficient condition is given so that these operators, in general unbounded, are commuting and selfadjoint. The spectral theorem for these operators is used to establish the existence of the measure of orthogonality in Favard's theorem.
\end{abstract}

\section{INTRODUCTION}

Let $\mathbb{N}_{0}$ be the set of nonnegative integers. For $\alpha=\left(\alpha_{1}, \ldots, \alpha_{d}\right) \in \mathbb{N}_{0}^{d}$ and $\mathbf{x}=\left(x_{1}, \ldots, x_{d}\right) \in \mathbb{R}^{d}$ we use the standard notation $\mathbf{x}^{\alpha}=x_{1}^{\alpha_{1}} \cdots x_{d}^{\alpha_{d}}$. The number $|\alpha|=\alpha_{1}+\cdots+\alpha_{d} \in \mathbb{N}_{0}$ is called the total degree of $\mathbf{x}^{\alpha}$. For $n \in \mathbb{N}_{0}$ we denote by $\Pi_{n}^{d}$ the set of polynomials of total degree at most $n$ in $d$ variables, and $\Pi^{d}$ the set of all polynomials in $d$ variables.

Let $\mathscr{L}$ be a linear functional defined on $\Pi^{d}$ such that $\mathscr{L}\left(g^{2}\right)>0$ whenever $g \neq 0$. Such an $\mathscr{L}$ is called square positive, it induces an inner product on $\Pi^{d}$. Thus, the Gram-Schmidt orthogonalization process can be applied to $\left\{x^{\alpha}\right\}$ to obtain a system of multivariate orthonormal polynomials. These polynomials share many properties of the univariant orthogonal polynomials. In particular, they satisfy three-term relations that now take vector-matrix form. Let $r_{k}^{d}=$ $\operatorname{dim} \Pi_{k}^{d}-\operatorname{dim} \Pi_{k-1}^{d}=\left(\begin{array}{c}k+d-1 \\ k\end{array}\right)$. For a sequence of polynomials $\left\{P_{j}^{k}\right\}_{j=1}^{r_{k}^{d}}$, where the superscript $k$ means that $P_{j}^{k}$ is of total degree $k$, we use vector notation

$$
\mathbb{P}_{k}(\mathbf{x})=\left[P_{1}^{k}(\mathbf{x}), P_{2}^{k}(\mathbf{x}), \ldots, P_{r_{k}}^{k}(\mathbf{x})\right]^{T},
$$

where $r_{k}=r_{k}^{d}$. That $\left\{P_{j}^{k}\right\}_{j=1}^{r_{k}} \underset{k=0}{\infty}$ is orthonormal with respect to $\mathscr{L}$ is equivalent to $\mathscr{L}\left(\mathbb{P}_{n} \mathbb{P}_{m}^{T}\right)=\delta_{m, n} I$, where $I: r_{n}^{d} \times r_{n}^{d}$ is the identity matrix. The notation $A: i \times j$ means that $A$ is a matrix of size $i \times j$. We shall call $\left\{\mathbb{P}_{n}\right\}$ orthonormal polynomials for convenience. In $[14,15]$, we prove the following theorem.

Theorem 1. Let $\left\{\mathbb{P}_{k}\right\}_{k=0}^{\infty}, \mathbb{P}_{0} \neq 0$, be a sequence in $\Pi^{d}$. Then the following statements are equivalent:

Received by the editors April 1, 1992.

1991 Mathematics Subject Classification. Primary 33C50, 47B15.

Key words and phrases. Multivariate orthogonal polynomials, recurrence relation, commuting selfadjoint operators, determinate measure. 
(1) There exists a linear functional which is square positive on $\Pi^{d}$ and makes $\left\{\mathbb{P}_{k}\right\}_{k=0}^{\infty}$ an orthonormal basis in $\Pi^{d}$.

(2) For $k \geq 0,1 \leq i \leq d$, there exist matrices $A_{k, i}: r_{k}^{d} \times r_{k+1}^{d}$ and $B_{k, i}: r_{k}^{d} \times$ $r_{k}^{d}$, such that

(a) $x_{i} \mathbb{P}_{k}=A_{k, i} \mathbb{P}_{k+1}+B_{k, i} \mathbb{P}_{k}+A_{k-1, i}^{T} \mathbb{P}_{k-1}, 1 \leq i \leq d$, and

(b) $\operatorname{rank} A_{k}=r_{k+1}^{d}$,

where $A_{-1, i}$ is taken to be zero.

This theorem extends Favard's theorem for the univariant case (cf. [3, p. 21]). The vector-matrix equation in (2) is the analogue of the three-term relation. We shall write $A_{n, 1}=a_{n}$ for $d=1$. For practical purposes, for example in the Gaussian cubature formula, it is very important to know whether $\mathscr{L}$ in Theorem 1 has an integral representation with respect to a nonnegative Borel measure-measure of orthogonality. For the univariant case it is well known that such a measure always exists. Moreover, if Carleman's condition $\sum\left(1 / a_{n}\right)=\infty$ holds, the measure is unique. However, since it is known that $\mathscr{L}\left(g^{2}\right)>0$ is not equivalent to $\mathscr{L}(g)>0$ for $g>0$, unless $d=1$, it follows from the theory of the moment problem (cf. $[2,5])$ that there may not exist such a measure of orthogonality for $d>1$. In [15] we proved that if the coefficient matrices in the three-term relation are uniformly bounded in spectral norm, then there is a unique measure. The purpose of this paper is to study the unbounded case. Our result shows that an analogy of Carleman's condition is sufficient for the existence and uniqueness of the measure of orthogonality.

We use an approach that is based on the operator theory. The connection between univariant orthogonal polynomials and the Jacobi matrix as an operator on $l^{2}$ is well known (cf. [1,13]). For multivariate orthonormal polynomials, we define associated operators as follows. Let $\mathscr{L}$ be a square positive linear functional, and let $\left\{\mathbb{P}_{n}\right\}_{n=0}^{\infty}$ be a sequence of orthonormal polynomials satisfying the three-term relation in Theorem 1 . Then linear operators $T_{i}, 1 \leq i \leq d$, are defined to be matrix operators.

$$
T_{i}=\left[\begin{array}{cccc}
B_{0, i} & A_{0, i} & & 0 \\
A_{0, i}^{T} & B_{1, i} & A_{1, i} & \\
& A_{1, i}^{T} & B_{2, i} & \ddots \\
0 & & \ddots & \ddots
\end{array}\right], \quad 1 \leq i \leq d,
$$

which act via matrix multiplication on $l^{2}$. The domain of $T_{i}$ consists of all sequences in $l^{2}$ for which matrix multiplication yields a sequence in $l^{2}$. The connection between these operators and $\left\{\mathbb{P}_{n}\right\}$ can be seen as follows. Let $\left\{\psi_{n}\right\}_{n=0}^{\infty}$ be the canonical orthonormal basis for $l^{2}$. We rewrite this basis as $\left\{\psi_{n}\right\}_{n=0}^{\infty}=\left\{\phi_{j}^{k}\right\}_{j=1}^{r_{k}}{\underset{k=0}{\infty}}^{\infty}$ according to the lexicographical order, and introduce the formal vector notation

$$
\Phi_{k}=\left[\phi_{1}^{k}, \ldots, \phi_{r_{k}}^{k}\right]^{T}, \quad k \in \mathbb{N}_{0} .
$$

The orthogonality of $\left\{\psi_{n}\right\}_{n=0}^{\infty}$ can be described as

$$
\left\langle\Phi_{k} \Phi_{m}^{T}\right\rangle=\left(\left\langle\phi_{i}^{k}, \phi_{j}^{m}\right\rangle\right)_{i=1}^{r_{k}}{ }_{j=1}^{r_{m}}=\delta_{i j} \delta_{k m} I
$$


We shall say that $\left\{\Phi_{n}\right\}_{n=0}^{\infty}$ is orthonormal. Let $\mathscr{P}$ be the space of $\Pi^{d}$ equipped with the inner product induced by $\mathscr{L}$ and the orthonormal basis $\left\{\mathbb{P}_{n}\right\}$. We define multiplication operators $\Lambda_{1}, \ldots, \Lambda_{d}$ on $\mathscr{P}$ by $\Lambda_{i} P=x_{i} P$. Let $U$ be a map from $\mathscr{P}$ into $l^{2}$ defined by $U: \mathbb{P}_{n} \mapsto \Phi_{n}$. Then $U$ is a unitary map. The three-term relation implies that the multiplication operators $\Lambda_{1}, \ldots, \Lambda_{d}$ are transformed into operators given by the matrices $T_{1}, \ldots, T_{d}$.

For $d=1$ we have only one operator, whose matrix representation is the classical Jacobi matrix. We call the matrices $T_{i}$ block Jacobi matrices. The elements of $T_{i}$ are matrices whose sizes increase when moving down the main diagonal. If these operators are selfadjoint and commuting, then the spectral theorem for a commuting family of operators can be applied to establish the existence of the measure. In [15] we considered the bounded case. Since the fact that operators commute means their spectral measures commute, there is a significant difference between bounded and unbounded cases (cf. [7]). We describe our results in $\S 2$ and prove them in $\S 3$.

\section{MAin Results}

Let $\mathscr{M}=\mathscr{M}\left(\mathbb{R}^{d}\right)$ denote the set of nonnegative Borel measures $\mu$ on $\mathbb{R}^{d}$, such that

$$
\int_{\mathbb{R}^{d}}\left|\mathbf{x}^{\alpha}\right| d \mu(\mathbf{x})<+\infty, \quad \forall \alpha \in \mathbb{N}_{0}^{d} .
$$

For $\mu \in \mathscr{M}$ the numbers $\mu_{\alpha}=\int \mathbf{x}^{\alpha} d \mu(\mathbf{x}), \alpha \in \mathbb{N}_{0}^{d}$, are called the moments of $\mu$. We are interested in the case for which $\mathscr{L}$ has a unique integral representation

$$
\mathscr{L}(f)=\int_{\mathbb{R}^{d}} f(\mathbf{x}) d \mu(\mathbf{x}), \quad \mu \in \mathscr{M} .
$$

The uniqueness of such a representation is in terms of the determinacy of the measure. Two measures are called equivalent if they have the same moments. The measure $\mu$ is called determinate if the equivalent class of measures having the same moments as $\mu$ consists of $\mu$ only.

Let $\|\cdot\|_{2}$ be the spectral norm for matrices that is induced by the Euclidean norm for vectors:

$$
\|A\|_{2}=\max \left\{\sqrt{\lambda}: \lambda \text { is an eigenvalue of } A^{T} A\right\} .
$$

In [15], we proved that $T_{i}$ is bounded if and only if $\left\{\left\|A_{k, i}\right\|_{2}\right\}$ and $\left\{\left\|B_{k, i}\right\|_{2}\right\}$ are bounded. We now state our main result.

Theorem 2. Let $\left\{\mathbb{P}_{n}\right\}_{n=0}^{\infty}, \mathbb{P}_{0} \neq 0$, be a sequence in $\Pi^{d}$ that satisfies the threeterm relation and rank condition in (2). If

$$
\sum_{k=0}^{\infty} \frac{1}{\left\|A_{n, i}\right\|_{2}}=\infty, \quad 1 \leq i \leq d,
$$

then there exists a determinate measure $\mu \in \mathscr{M}$ such that $\left\{\mathbb{P}_{n}\right\}$ is orthonormal with respect to $\mu$.

This theorem extends the result in [15] by allowing the unbounded cases. A typical example is the product of Hermite polynomials. In the univariant case the condition $(*)$ is well known (cf. $[1$, p. 24; 4]). The classical result of Carleman on the determinacy in moment problem [1, p. 86] follows from 
this condition. For $n \in \mathbb{N}_{0}$ let $\mu_{n}=\mathscr{L}\left(x^{n}\right)$ for $\mathscr{L}$ defined on $\Pi^{1}$. Carleman's result says that $\left\{\mu_{n}\right\}$ is determinate if it satisfies Carleman's condition $\sum_{n=0}^{\infty}\left(\mu_{2 n}\right)^{-1 / 2 n}=\infty$. This result was extended by Nussbaum [8] to the multivariate moment problem. Let $\mu_{\alpha}=\mathscr{L}\left(\mathbf{x}^{\alpha}\right)$ for $\mathscr{L}$ on $\Pi^{d}$ and denote by $\mu_{n, i}$ the marginal sequences $\mu_{n, i}=\mathscr{L}\left(x_{i}^{n}\right), 1 \leq i \leq d$. Then Naussbaum's result says that $\left\{\mu_{\alpha}\right\}$ is determinate if $\left\{\mu_{n, i}\right\}$ verifies Carleman's condition. The proof of this result in [8] is based on the theory of quasi-analytic vectors. A natural question is then whether Nussbaum's result follows from condition $(*)$ in Theorem 2 . This is discussed in the following.

For $n \in \mathbb{N}_{0}$ and $\alpha \in \mathbb{N}_{0}^{d}$, we denote by $\mathbf{x}^{n}$ the vector $\left\{\mathbf{x}^{\alpha}\right\}_{|\alpha|=n}$ where the elements are numbered according to the lexicographical order in $\left\{\alpha \in \mathbb{N}_{0},|\alpha|=\right.$ $n$ \}. The orthonormal polynomials $\mathbb{P}_{n}$ with respect to $\mathscr{L}$ can be written as $\mathbb{P}_{n}=G_{n} \mathbf{x}^{n}+\mathbb{Q}_{n}$, where $\mathbb{Q}_{n}$ is a polynomial vector with components in $\Pi_{n-1}^{d}$. In [16] we proved that the matrix $G_{n}$ is invertible. Let $L_{n, i}$ denote the matrices of size $r_{n-1}^{d} \times r_{n}^{d}$ satisfying $L_{n, i} \mathbf{x}^{n}=x_{i} \mathbf{x}^{n-1}$. Since $x_{i}^{n}=L_{1, i} \cdots L_{n, i} \mathbf{x}^{n}$, we have

$$
\mathscr{L}\left(x_{i}^{n} \mathbb{P}_{n}^{T}\right)=L_{1, i} \cdots L_{n, i} \mathscr{L}\left(\mathbf{x}^{n} \mathbb{P}_{n}^{T}\right)=L_{1, i} \cdots L_{n, i} G_{n}^{-1} \mathscr{L}\left(\mathbb{P}_{n} \mathbb{P}_{n}^{T}\right) .
$$

Therefore, from equation $A_{n, i} G_{n+1}=G_{n} L_{n+1, i}$ [16], we have $\mathscr{L}\left(\mathbb{P}_{0}\right)=G_{0}$ $(=1$, say $)$ and

$$
\mathscr{L}\left(x_{i}^{n} \mathbb{P}_{n}^{T}\right)=A_{0, i} \cdots A_{n-1, i}, \quad n \geq 1 .
$$

On the other hand, $\mathscr{L}$ induces an inner product on $\Pi^{d}$ by $\langle f, g\rangle=\mathscr{L}(f g)$. Thus from Cauchy's inequality in the inner product space we have

$$
\left\|\mathscr{L}\left(x_{i}^{n} \mathbb{P}_{n}^{T}\right)\right\|_{2}^{2} \leq \mathscr{L}\left(x_{i}^{2 n}\right) \mathscr{L}\left(\mathbb{P}_{n} \mathbb{P}_{n}^{T}\right)=\mu_{2 n, i}
$$

It then follows that $\left\|A_{0, i} \cdots A_{n-1, i}\right\|_{2}^{2} \leq \mu_{2 n, i}$. From a general inequality of Carleman (cf. [1, p. 86])

$$
\sum_{n=0}^{\infty}\left(u_{1} u_{2} \cdots u_{n}\right)^{1 / n}<e \sum_{n=0}^{\infty} u_{n},
$$

where the $u_{k}$ are nonnegative real numbers not all of which are zero, it then follows that

$$
\sum_{n=1}^{\infty}\left(\mu_{2 n, i}\right)^{-1 / 2 n} \leq \sum_{n=1}^{\infty} \frac{1}{\left(\left\|A_{0, i} \cdots A_{n-1, i}\right\|_{2}\right)^{1 / n}} \leq e \sum_{n=1}^{\infty} \frac{\left\|A_{0, i} \cdots A_{n-2, i}\right\|_{2}}{\left\|A_{0, i} \cdots A_{n-1, i}\right\|_{2}} .
$$

Therefore, Nussbaum's condition implies

$$
\sum_{n=0}^{\infty} \frac{\left\|A_{0, i} \cdots A_{n-1, i}\right\|_{2}}{\left\|A_{0, i} \cdots A_{n, i}\right\|_{2}}=\infty, \quad 1 \leq i \leq d .
$$

Unfortunately, this condition does not match our condition $(*)$ unless $d=1$, in which case the matrices become numbers.

Clearly, both conditions $(*)$ and $(* *)$ can be viewed as natural extensions of the univariant condition $\sum\left(1 / a_{n}\right)=\infty$. This also applies to the condition

$$
\sum_{k=0}^{\infty}\left\|A_{n, i}^{\dagger}\right\|_{2}=\infty
$$


where $A_{n, i}^{\dagger}$ denotes the generalized inverse of $A_{n, i}$. Since $A_{n, i}$, as the coefficient of the three-term relation, is necessarily of full rank, $\operatorname{rank} A_{n, i}=r_{n}^{d}$, the generalized inverse exists. We note that $(*)$ implies $(* *)$, and $(* *)$ implies $(\dagger)$. An open question is whether Theorem 2 can be strengthened to hold under condition $(* *)$, or even $(\dagger)$. If the answer is yes, then the above discussion gives another proof of Nussbaum's result.

\section{PROOFS}

First we recall the part of spectral theory that will be needed (see $[10,11$, 12]). Let $\mathscr{H}$ be a separable Hilbert space and $T_{i}: \mathscr{H} \mapsto \mathscr{H}$ be selfadjoint operators. Let $E_{i}$ be the spectral measure of $T_{i}, T_{i}=\int x d E_{i}(x)$, which is a projection-valued measure defined for Borel sets of $\mathbb{R}$ such that $E_{i}(\mathbb{R})$ is the identity operator in $\mathscr{H}$ and $E_{i}(B \cap C)=E_{i}(B) \cap E_{i}(C)$ for Borel sets $B, C \subseteq \mathbb{R}$. The fact that $\left\{T_{1}, \ldots, T_{d}\right\}$ commute means that their spectral measure commutes, i.e., $E_{i}(B) E_{j}(C)=E_{i}(C) E_{j}(B)$ for any $i, j=1, \ldots, d$ and any two Borel sets $B, C \subseteq \mathbb{R}$. If $T_{1}, \ldots, T_{d}$ commute, then

$$
E=E_{1} \otimes \cdots \otimes E_{d}
$$

is a spectral measure on $\mathbb{R}^{d}$ with values that are selfadjoint projections in $\mathscr{H}$. In particular, $E$ is the unique measure such that

$$
E\left(B_{1} \times \cdots \times B_{d}\right)=E_{1}\left(B_{1}\right) \cdots E_{d}\left(B_{d}\right)
$$

for any Borel sets $B_{1}, \ldots, B_{d} \subseteq \mathbb{R}$. The measure $E$ is called the spectral measure of the commuting family $T_{1}, \ldots, T_{d}$. A vector $\Phi_{0} \in \mathscr{H}$ is a cyclic vector in $\mathscr{H}$ with respect to the commuting family of selfadjoint operators $T_{1}, \ldots, T_{d}$ in $\mathscr{H}$ if the linear manifold $\left\{P\left(T_{1}, \ldots, T_{d}\right) \Phi_{0}, P \in \Pi^{d}\right\}$ is dense in $\mathscr{H}$. The spectral theorem for $T_{1}, \ldots, T_{d}$ is as follows.

Theorem 3. Let $\mathscr{H}$ be a separable Hilbert space and $T_{1}, \ldots, T_{d}$ be a commuting family of selfadjoint operators in $\mathscr{H}$. If $\Phi_{0}$ is a cyclic vector in $\mathscr{H}$ with respect to $T_{1}, \ldots, T_{d}$, then $T_{1}, \ldots, T_{d}$ are unitarily equivalent to the multiplication operators $X_{1}, \ldots, X_{d}$,

$$
\left(X_{i} f\right)(\mathbf{x})=x_{i} f(\mathbf{x}), \quad 1 \leq i \leq d,
$$

defined on $L^{2}\left(\mathbb{R}^{d}, \mu\right)$, where the measure $\mu$ is defined by $\mu(B)=\left\langle E(B) \Phi_{0}, \Phi_{0}\right\rangle$ for the Borel set $B \subset \mathbb{R}^{d}$.

The unitary equivalence means that there exists a unitary mapping $U: \mathscr{H} \rightarrow$ $L^{2}\left(\mathbb{R}^{d}, \mu\right)$ such that $U T_{i} U^{-1}=X_{i}, 1 \leq i \leq d$. The unitary equivalence in Theorem 3 associates the cyclic vector $\bar{\Phi}_{0}$ with the function $f(\mathbf{x})=1$ and $\left(T_{1}^{\alpha_{1}} \cdots T_{d}^{\alpha_{d}}\right) \Phi_{0}$ with $f(\mathbf{x})=\mathbf{x}^{\alpha}$.

We need to show that if condition $(*)$ is satisfied, then the operators $T_{1}, \ldots, T_{d}$ defined in equation (2) form a commuting family of selfadjoint operators that has a cyclic vector.

Let $\mathscr{D}(T)$ denote the domain of the operator $T, \mathscr{D}(T)=\{f: T f \in \mathscr{H}\}$. From now on we set $\mathscr{H}=l^{2}$. We denote by $\mathscr{D}$ the set of all finite linear combinations of canonical basis vectors, or in our vector notation $\Phi_{k}$,

$$
\mathscr{D}=\left\{\sum_{k=0}^{N} \mathbf{a}_{k}^{T} \Phi_{k}: N \in \mathbb{N}_{0}, \mathbf{a}_{k} \in \mathbb{R}^{r_{k}}\right\} .
$$


Clearly $\mathscr{D}$ is a dense subset of $\mathscr{H}$. For any $f$ in $\mathscr{D}\left(T_{i}\right), f=\sum \mathbf{a}_{k}^{T} \boldsymbol{\Phi}_{k}$, we have from the definition that

$$
T_{i} f=\sum_{k=0}^{\infty} \mathbf{a}_{k}^{T}\left[A_{k, i} \boldsymbol{\Phi}_{k+1}+B_{k, i} \boldsymbol{\Phi}_{k}+A_{k-1, i}^{T} \boldsymbol{\Phi}_{k-1}\right] .
$$

Therefore, $\mathscr{D} \subset \mathscr{D}\left(T_{i}\right)$, and it follows that $T_{i}$ is densely defined on $\mathscr{H}$.

Lemma 1. If condition $(*)$ is satisfied then the operator $T_{i}$ defined in equation (2) is selfadjoint.

Proof. Let $f, g \in \mathscr{D}\left(T_{i}\right), f=\sum \mathbf{a}_{k}^{T} \Phi_{k}$ and $g=\sum \mathbf{b}_{k}^{T} \Phi_{k}$. First we prove that $T_{i}$ is symmetric, i.e., $\left\langle T_{i} f, g\right\rangle=\left\langle T_{i} g, f\right\rangle$. The orthogonality of $\mathbb{P}_{n}$ implies that the $B_{n, i}$ are symmetric matrices, $B_{n, i}=\mathscr{L}\left(x_{i} \mathbb{P}_{n} \mathbb{P}_{n}^{T}\right)$. From the definition of $T_{i}$ we have

$$
\left\langle T_{i} f, g\right\rangle=\lim _{n \rightarrow \infty} S_{n}\left(\left\langle T_{i} f, g\right\rangle\right),
$$

where

$$
\begin{aligned}
S_{n}\left(\left\langle T_{i} f, g\right\rangle\right) & =\sum_{k=0}^{n}\left[\mathbf{a}_{k-1}^{T} A_{k-1, i}+\mathbf{a}_{k}^{T} B_{k, i}+\mathbf{a}_{k+1}^{T} A_{k, i}^{T}\right] \mathbf{b}_{k} \\
& =\sum_{k=0}^{n} \mathbf{b}_{k}^{T}\left[A_{k-1, i}^{T} \mathbf{a}_{k-1}+B_{k, i} \mathbf{a}_{k}+A_{k, i} \mathbf{a}_{k+1}\right] \\
& =\sum_{k=0}^{n-1} \mathbf{b}_{k+1}^{T} A_{k, i}^{T} \mathbf{a}_{k}+\sum_{k=0}^{n} \mathbf{b}_{k}^{T} B_{k, i} \mathbf{a}_{k}+\sum_{k=1}^{n+1} \mathbf{b}_{k-1}^{T} A_{k-1, i} \mathbf{a}_{k} .
\end{aligned}
$$

Therefore, it follows readily that

$$
\begin{aligned}
& \left|S_{n}\left(\left\langle T_{i} f, g\right\rangle\right)-S_{n}\left(\left\langle T_{i} g, f\right\rangle\right)\right| \\
& \quad=\left|\mathbf{b}_{n}^{T} A_{n, i} \mathbf{a}_{n+1}-\mathbf{a}_{n}^{T} A_{n, i} \mathbf{b}_{n+1}\right| \\
& \quad \leq\left\|A_{n, i}\right\|_{2}\left(\left\|\mathbf{b}_{n}\right\|_{2}\left\|\mathbf{a}_{n+1}\right\|_{2}+\left\|\mathbf{a}_{n}\right\|_{2}\left\|\mathbf{b}_{n+1}\right\|_{2}\right) \\
& \quad \leq\left\|A_{n, i}\right\|_{2}\left(\left\|\mathbf{b}_{n}\right\|_{2}^{2}+\left\|\mathbf{a}_{n+1}\right\|_{2}^{2}+\left\|\mathbf{a}_{n}\right\|_{2}^{2}+\left\|\mathbf{b}_{n+1}\right\|_{2}^{2}\right) / 2 .
\end{aligned}
$$

If $\left\langle T_{i} f, g\right\rangle-\left\langle T_{i} g, f\right\rangle=\delta$, then for a sufficiently large $N$ we have

$$
\frac{\delta}{2} \sum_{n \geq N} \frac{1}{\left\|A_{n, i}\right\|_{2}} \leq \sum\left\|\mathbf{b}_{n}\right\|_{2}^{2}+\sum\left\|\mathbf{a}_{n}\right\|_{2}^{2} \leq\|f\|^{2}+\|g\|^{2} .
$$

Therefore, our condition $(*)$ implies that $\delta=0$. Thus, $T_{i}$ is symmetric. We now prove that $T_{i}$ is selfadjoint, i.e., $\left.T_{i}\right|_{\mathscr{D}\left(T_{i}^{*}\right)}=T_{i}^{*}$. Assume $g \in \mathscr{D}\left(T_{i}^{*}\right)$ and $T^{*} g=f$. Let again $f=\sum \mathbf{a}_{k}^{T} \Phi_{k}$ and $g=\sum \mathbf{b}_{k}^{T} \Phi_{k}$. Since

$$
\left\langle T_{i} \Phi_{k}, g\right\rangle=A_{k, i} \mathbf{b}_{k+1}+B_{k, i} \mathbf{b}_{k}+A_{k-1, i}^{T} \mathbf{b}_{k-1}
$$

it follows from $\left\langle T_{i} \Phi_{k}, g\right\rangle=\left\langle\Phi_{k}, T_{i}^{*} g\right\rangle$ that $\mathbf{a}_{k}=A_{k, i} \mathbf{b}_{k+1}+B_{k, i} \mathbf{b}_{k}+$ $A_{k-1, i}^{T} \mathbf{b}_{k-1}$. Therefore,

$$
\begin{aligned}
f & =\sum \mathbf{a}_{k}^{T} \boldsymbol{\Phi}_{k}=\sum\left[A_{k, i} \mathbf{b}_{k+1}+B_{k, i} \mathbf{b}_{k}+A_{k-1, i}^{T} \mathbf{b}_{k-1}\right]^{T} \boldsymbol{\Phi}_{k} \\
& =\sum \mathbf{b}_{k}^{T}\left[A_{k, i} \boldsymbol{\Phi}_{k+1}+B_{k, i} \boldsymbol{\Phi}_{k}+A_{k-1, i}^{T} \boldsymbol{\Phi}_{k-1}\right]=T_{i} g .
\end{aligned}
$$

That is, $T_{i}^{*} g=T_{i} g$ for all $g \in \mathscr{D}^{*}\left(T_{i}\right)$. Thus, $T_{i}$ is selfadjoint. 
For the proof of Theorem 2 we only need $T_{i}$ to be essentially selfadjoint and their closures to commute. The essential selfadjointness of $T_{i}$ can also be derived from [6]. For bounded operators, the fact that $T_{i}$ and $T_{j}$ have commuting spectral measures is equivalent to $T_{i} T_{j}=T_{j} T_{i}$. But for unbounded operators this is no longer true (cf. [7]). We need the following result due to Nelson [7] (see also [9]).

Lemma 2. Let $T$ and $S$ be symmetric operators in a Hilbert space $\mathscr{H}$ and let $D$ be a dense linear manifold in $\mathscr{H}$ such that $D$ is contained in the domain of $T^{2}, S^{2}, T S$, and $S T$, and such that $T S f=S T f$ for all $f$ in $\mathscr{H}$. If the restriction of $S^{2}+T^{2}$ to $D$ is essentially selfadjoint then $T$ and $S$ are essentially selfadjoint and $\bar{S}$ and $\bar{T}$ commute, where $\bar{T}$ stands for the closure of $T$.

Lemma 3. If condition (*) is satisfied then the operators $T_{1}, \ldots, T_{d}$ defined in equation (2) mutually commute.

Proof. Since our $T_{i}$ is selfadjoint by the previous lemma, $\bar{T}_{i}=T_{i}^{* *}=T_{i}$. The orthogonality of $\mathbb{P}_{n}$ in Theorem 1 implies that the coefficient matrices satisfy [14]

$$
\begin{gathered}
A_{k, i} A_{k+1, j}=A_{k, j} A_{k+1, i}, \\
A_{k, i} B_{k+1, j}+B_{k, i} A_{k, j}=B_{k, j} A_{k, i}+A_{k, j} B_{k+1, i}, \\
A_{k-1, i}^{T} A_{k-1, j}+B_{k, i} B_{k, j}+A_{k, i} A_{k, j}^{T}=A_{k-1, j}^{T} A_{k-1, i}+B_{k, i} B_{k, j}+A_{k, j} A_{k, i}^{T},
\end{gathered}
$$

for $i \neq j, 1 \leq i, j \leq d$, and $k \geq 0$, where $A_{-1, i}=0$. Using these equations it is readily seen that $T_{i} T_{j} f=T_{j} T_{i} f$ on $\mathscr{D}\left(T_{i} T_{j}\right) \cap \mathscr{D}\left(T_{j} T_{i}\right)$. In particular, $T_{i} T_{j} f=T_{j} T_{i} f$ for all $f$ in $\mathscr{D}$, where $\mathscr{D}$ contains all finite linear combinations of $\Phi_{k}$ as defined before. Since $\mathscr{D}$ is a dense linear manifold in $\mathscr{H}$ and clearly $\mathscr{D}$ is contained in $\mathscr{D}\left(T_{i}^{2}\right), \mathscr{D}\left(T_{j}^{2}\right), \mathscr{D}\left(T_{i} T_{j}\right)$, and $\mathscr{D}\left(T_{j} T_{i}\right)$, we can apply Lemma 2. For $i \neq j$ let $T_{i j}$ be the restriction of $T_{i}^{2}+T_{j}^{2}$ on $\mathscr{D}$. If condition $(*)$ holds, then the selfadjointness of $T_{i}$ and $T_{j}$ by Lemma 1 implies that

$$
\left\langle\left(T_{i}^{2}+T_{j}^{2}\right) f, g\right\rangle=\left\langle f,\left(T_{i}^{2}+T_{j}^{2}\right) g\right\rangle
$$

for all $f, g$ in $\mathscr{D}$. Thus, $T_{i j}$ is symmetric. Assume that $g \in \mathscr{D}\left(T_{i j}^{*}\right)$ and $f=T_{i j}^{*} g$, and write $f=\sum \mathbf{a}^{T} \Phi_{k}$ and $g=\sum \mathbf{b}_{k}^{T} \Phi_{k}$. First we have

$$
T_{i, j} \boldsymbol{\Phi}_{k}=C_{k+1} \boldsymbol{\Phi}_{k+2}+D_{k+1} \boldsymbol{\Phi}_{k+1}+E_{k} \boldsymbol{\Phi}_{k}+D_{k}^{T} \boldsymbol{\Phi}_{k-1}+C_{k-1}^{T} \Phi_{k-2},
$$

where

$$
\begin{aligned}
C_{k+1}= & A_{k, i} A_{k+1, i}+A_{k, j} A_{k+1, j}, \\
D_{k+1}= & A_{k, i} B_{k+1, i}+B_{k, i} A_{k, i}+A_{k, j} B_{k+1, j}+B_{k, j} A_{k+1, j}, \\
E_{k}= & A_{k, i} A_{k, i}^{T}+B_{k, i} B_{k, i}+A_{k-1, i}^{T} A_{k-1, i} \\
& +A_{k, j} A_{k, j}^{T}+B_{k, j} B_{k, j}+A_{k-1, j}^{T} A_{k-1, j} .
\end{aligned}
$$

Then as in the proof of Lemma 1 , we can compute $\left\langle T_{i j} \Phi_{k}, g\right\rangle$ and use $\left\langle\Phi_{k}, T_{i j}^{*} g\right\rangle=\left\langle T_{i j} \Phi_{k}, g\right\rangle$ to write $\mathbf{a}_{k}$ in terms of $\left\{\mathbf{b}_{k}\right\}$. Thus we obtain

$$
\mathbf{a}_{k}=C_{k+1} \mathbf{b}_{k+2}+D_{k+1} \mathbf{b}_{k+1}+E_{k} \mathbf{b}_{k}+D_{k}^{T} \mathbf{b}_{k-1}+C_{k-1}^{T} \mathbf{b}_{k-2} .
$$


Using this relation in $f=\sum \mathbf{a}_{k}^{T} \Phi_{k}$ and changing the summation variables, we obtain

$$
f=\sum \mathbf{a}_{k}^{T} \Phi_{k}=\sum \mathbf{b}_{k}^{T} T_{i, j} \Phi_{k}=T_{i j} g .
$$

Thus, $T_{i j}$ is selfadjoint. From Lemma 2 it follows that $T_{i}$ and $T_{j}$ commute.

The next lemma is proved in [15] for bounded operators, but its proof does not depend on the boundedness of $T_{i}$.

Lemma 4. The vector $\Phi_{0} \in \mathscr{H}$ is a cyclic vector with respect to $T_{1}, \ldots, T_{d}$ and

$$
\Phi_{n}=\mathbb{P}_{n}\left(T_{1}, \ldots, T_{d}\right) \Phi_{0},
$$

where $\mathbb{P}_{n}\left(x_{1}, \ldots, x_{d}\right)$ is of the form used in equation (1).

Proof of Theorem 2. The proof follows the same line as in the bounded case [15]; we shall be brief. Let $\left\{\mathbb{P}_{n}\right\}$ satisfy the three-term relation in the theorem. If condition $(*)$ is satisfied, then it follows from previous lemmas and Theorem 3 that $T_{1}, \ldots, T_{d}$ are unitarily equivalent to the multiplication operators $X_{1}, \ldots, X_{d}$ in $L^{2}\left(\mathbb{R}^{d}, \mu\right)$, where the measure $\mu \in \mathscr{M}$ is defined by $\mu(B)=\left\langle E(B) \Phi_{0}, \Phi_{0}\right\rangle$. The polynomials $\left\{\mathbb{P}_{n}\right\}$ in Lemma 4 are the polynomials that satisfy the three-term relation, they are orthonormal with respect to $\mu$ as

$$
\int \mathbb{P}_{n}(\mathbf{x}) \mathbb{P}_{m}^{T}(\mathbf{x}) d \mu(\mathbf{x})=\left\langle\mathbb{P}_{n} \Phi_{0}, \mathbb{P}_{m}^{T} \Phi_{0}\right\rangle=\left\langle\Phi_{n}, \Phi_{m}^{T}\right\rangle .
$$

Therefore, the existence of the measure of orthogonality is proved. Since the unitary equivalence implies that each of the multiplication operators $X_{j}$ is selfadjoint, the determinacy of $\mu$ follows from [5, Theorem 7].

\section{ACKNOWLEDGMENT}

The author thanks a referee for his helpful comments.

\section{REFERENCES}

1. N. I. Akheizer, The classical moment problem and some related questions in analysis, Hafner, New York, 1965.

2. C. Berg, The multidimensional moment problem and semigroups, Moments in Mathematics, Proc. Sympos. Appl. Math., vol. 37, Amer. Math. Soc., Providence, RI, 1987, pp. 110-124.

3. T. S. Chihara, An introduction to orthogonal polynomials, Math. Appl., vol. 13, Gordon \& Breach, New York, 1978.

4. J. Dombrowski, Cyclic operators, commutators, and absolutely continuous measures, Proc. Amer. Math. Soc. 100 (1987), 457-463.

5. B. Fuglede, The multidimensional moment problem, Exposition. Math. 1 (1983), 47-65.

6. P. E. T. Jorgensen, Essential self-adjointness of semibounded operators, Math. Ann. 237 (1978), 187-192.

7. E. Nelson, Analytic vectors, Ann. of Math. (2) 70 (1959), 572-615.

8. A. E. Nussbaum, Quasi-analytic vectors, Ark. Mat. 6 (1966), 179-191.

9. Math. Soc. 140 (1969), 485-491.

10. F. Riesz and B. Sz.-Nagy, Functional analysis, Ungar, New York, 1955.

11. Y. S. Samoilenko, Spectral theory of families of self-adjoint operators, Kluwer, The Netherlands, 1991; translated from Russian ed., Moscow, 1987. 
12. B. D. Sleeman, Multiparameter spectral theory in Hilbert spaces, Pitman, London, 1978.

13. M. Stone, Linear transformations in Hilbert space and their applications to analysis, Amer. Math. Soc. Colloq. Publ., vol. 15, Amer. Math. Soc., Providence, RI, 1932, reprinted 1983.

14. Y. Xu, On multivariate orthogonal polynomials, SIAM J. Math. Anal. 24 (1993).

15. __ Multivariate orthogonal polynomials and operator theory, Trans. Amer. Math. Soc. (to appear).

16. __ Recurrence formulas for multivariate orthogonal polynomials, Math. Comp. (1994) (to appear).

Department of Mathematics, University of Oregon, Eugene, Oregon 97403

E-mail address: yuan@bright.uoregon.edu 